\title{
Cultivo de feijão-vagem em vaso em diferentes substratos e condutividades elétricas da solução nutritiva
}

\section{Bean-pod cultivation in pots with different substrates and electrical conductivity of nutrient solution}

\author{
José Marcelo Basso ${ }^{1 *}$; Hideaki Wilson Takahashi²; \\ Gustavo Adolfo de Freitas Fregonezi ${ }^{2}$
}

\begin{abstract}
Resumo
A exigência dos consumidores por produtos mais saudáveis, aliada a problemas como salinização, doenças de solo e cultivos sucessivos, promovem a busca por sistemas produtivos mais sustentáveis que os atuais. Assim o presente trabalho teve como objetivo a avaliação nutricional das plantas em função da condutividade elétrica da solução nutritiva utilizando diferentes substratos. $O$ experimento foi conduzido no Centro de Ciências Agrárias da UEL. Utilizou-se a cultivar UEL-2 de feijão-vagem, que possui crescimento determinado, altura média de $40 \mathrm{~cm}$, vagens retas com secção achatada e colheita em torno de 55 dias do plantio. O delineamento experimental foi blocos casualizados com parcelas subdivididas, as parcelas foram compostas dos tratamentos com soluções nutritivas contendo: 0,$5 ; 1,0$; 1,5 e $2,0 \mathrm{mS} \mathrm{cm}{ }^{-1}$ de condutividade elétrica e as subparcelas compostas por substratos: casca de arroz carbonizada; casca de Pinus e uma mistura composta de 50\% casca de arroz $+50 \%$ casca de Pinus. Foram utilizados 60 vasos de 5 litros, cada vaso com duas plantas, irrigados diariamente 2 vezes ao dia, com $150 \mathrm{ml}$ de solução nutritiva conforme sua condutividade elétrica do tratamento, com auxílio de um regador manual. Foram analisados os teores de matérias frescas e secas de raízes, caules, folhas e vagens, comprimento e diâmetro de vagens, condutividades elétricas e pH's do eluviado durante todo ciclo. Os resultados obtidos foram submetidos a analise de variância e as médias dos substratos comparadas pelo teste de Tukey a $5 \%$ de probabilidade, e as doses de Condutividade Elétrica por meio de regressão. $\mathrm{O}$ uso da solução nutritiva com condutividade elétrica de $1,5 \mathrm{mS} \mathrm{cm}^{-1}$ e o substrato com $50 \%$ de casca de pinus $+50 \%$ de casca de arroz carbonizada resultou em maior produtividade, com $182 \mathrm{~g} \mathrm{vaso}^{-1}$, correspondendo à $1,09 \mathrm{~kg} \mathrm{~m}^{2}$. Já a utilização do substrato arroz carbonizado com a condutividade elétrica de $0,5 \mathrm{mS} \mathrm{cm}^{-1}$, apresentou os piores resultados, com produção de $47,6 \mathrm{~g}$ vaso${ }^{1}$, sendo extrapolado para $0,286 \mathrm{~kg} \mathrm{~m}^{2}$.O substrato com a mistura de casca de arroz carbonizada mais casca de pínus, mostrou ser mais apropriado para ser utilizada como substrato. Dentre os tratamentos analisados, os melhores resultados foram obtidos com a utilização das maiores condutividades elétricas, entre 1,5 e $2,0 \mathrm{mS} \mathrm{cm}^{-1}$.

Palavras-chave: Phaseolus vulgaris, casca de pinus, casca de arroz carbonizada, fertirrigação, solução nutritiva
\end{abstract}

\begin{abstract}
The consumer's demand for healthier products along with issues such as salinization, soil affections and successive planting and cropping foment the pursuit for more sustainable production systems that the ones in practice currently. To such an extent, the present study aimed to evaluate the nutrition of plants
\end{abstract}

\footnotetext{
${ }^{1}$ Eng $^{\mathrm{o}} \mathrm{Agr}^{\mathrm{o}}$, Mestrando em Agronomia no Dept ${ }^{\mathrm{o}}$ de Agronomia, Universidade Estadual de Londrina, UEL, Londrina, PR. E-mail: josemarcelob@hotmail.com

${ }^{2}$ Profs. Drs. do Dept ${ }^{\circ}$ de Agronomia, UEL. Londrina, PR. E-mail: hwilson@uel.br; gustavofregonezi@yahoo.com.br

* Autor para correspondência
} 
due to electrical conductivity of nutrient using different substrates. The experiment was conducted at the Agrarian Sciences Center at UEL. For the cultivation was used UEL-2 bean pod type, which has determined growth, average height of $40 \mathrm{~cm}$, straight pods with flat section and harvest around 55 days after planting. The experiment outline was randomized blocks with subdivided plots. The plots were composed by treatments holding nutrient solutions with electrical conductivity of: $0.5,1.0,1.5$ and 2.0 $\mathrm{mS} \mathrm{cm}{ }^{-1}$ and subdivisions composed by substrates consisting of: carbonized rice hulls, Pinus bark and a combination of $50 \%$ carbonized rice hulls $+50 \%$ pine bark. A total of 60 pots of 5 liters each were used, each one holding two plants that were watered twice a day, with the aid of manual watering, containing $150 \mathrm{ml}$ of variable nutrient solution, depending on each of the treatments electrical conductivity. The levels of fresh and dry matter of roots, stems, leaves and pods, pod length and diameter, electrical conductivity and $\mathrm{pH}$ 's of eluviated ones were analyzed throughout the whole cycle. The obtained results were submitted to analysis of variance and the substrates averages compared by the Tukey test at $5 \%$ probability, and the quantities of electrical conductivity by means of regression. The use of nutrient solution with electrical conductivity of $1.5 \mathrm{mS} \mathrm{cm}^{-1}$ and the substrate containing $50 \%$ pine bark over $50 \%$ carbonized rice hulls resulted in increased productivity, with $182 \mathrm{~g} \mathrm{pot}^{-1}$, corresponding to $1.09 \mathrm{~kg}$ $\mathrm{m}^{2}$. At the same time, the use of carbonized rice hulls substrate with the electrical conductivity of 0.5 $\mathrm{mS} \mathrm{cm}-1$ showed the worst results, with production of $47.6 \mathrm{~g} \mathrm{pot}^{-1}$, and extrapolated to $0.286 \mathrm{~kg} \mathrm{~m}^{2}$. The substrate composed by the combination of carbonized rice hulls over pine bark was proved to be more suitable in substrate applications in such cases. Among the treatments tested, the best results were obtained using higher electrical conductivities between 1.5 and $2.0 \mathrm{mS} \mathrm{cm}$.

Key words: Phaseolus vulgaris, pine bark, carbonized rice hulls, fertilizing irrigation, nutrient solution

\section{Introdução}

O feijão-vagem (Phaseolus vulgaris L.) é a mais importante Fabacea no grupo das olerícolas. Difere do feijão comum porque suas vagens são colhidas ainda imaturas, sendo utilizadas na alimentação humana tanto de maneira industrializada como in natura (FILGUEIRA, 2000). Esta entre as hortaliças de maior comercialização, com produção média no Brasil em torno de 57 mil toneladas (IBGE, 2006). A comercialização é feita durante todos os meses do ano, sendo julho, agosto, setembro e outubro os meses de menor oferta do produto.

Assim a produção em ambiente protegido possibilita o cultivo nestes períodos de menor demanda, aumento os lucros do produtor. Podendo ser considera uma opção de rotação de cultura viável economicamente, importante para quebra de cultivos sucessivos, evitando assim inúmeras doenças de solos (podridão das raízes, mofo branco e murcha de fusarium) e problemas de ordem nutricional, como salinização do solo e/ou antagonismo entre nutrientes. Assim o emprego da fertirrigação pode reduzir problemas decorrente do cultivo em ambiente protegido e ser um ótima alternativa do cultivo do feijão-vagem (FERNANDES; CORÁ; ARAÚJO, 2001).

A fertirrigação tem despertado interesse, por reduzir os impactos ambientais. De modo geral, o aumento da produtividade, a maior eficiência na utilização de água de irrigação e fertilizantes, a redução da quantidade ou eliminação de alguns defensivos, e maior probabilidade de obtenção de produtos de qualidade são as principais vantagens dessa tecnologia (RODRIGUES, 2002). Para sua utilização, devem ser consideradas algumas características do substrato, como: favorecer a sustentação das plantas, possuírem boas condições físico-químicas, macroporosidade e microporosidade adequadas, drenagem, ausência de metais pesados, de fácil manuseio e transporte, além de preço compensador (MARTINEZ; BARBOSA, 1999; GONÇALVES; BENEDETTI, 2000).

A casca de arroz carbonizada, segundo Minami (1995) possui forma floculada, é leve, de fácil manuseio, com grande capacidade de drenagem, $\mathrm{pH}$ levemente alcalino, com baixa capacidade de retenção de umidade, rica em cálcio e potássio, livre de nematoides e patógenos devido ao processo de 
carbonização. Já a casca de pinus segundo Martin et al. (2006), é um material de fácil drenagem, baixa absorção de água, pH em torno de 3,7, de fácil obtenção por ser subproduto das indústrias de papel, contudo apresenta uma baixa concentração de nutrientes (SIMMLER et al., 1999). A mistura destes materiais um com materiais de elevada porosidade (Casca de Pinus) e o outro com boa capacidade de retenção de umidade (Casca de arroz Carbonizada) poderá trazer vantagens de cultivo.

Asolução nutritiva é o meio pelo qual os nutrientes previamente dissolvidos na água, são colocados à disposição das plantas, aumentando assim a rapidez de correção e eficiência do fertilizante (MARTINEZ; SILVA FILHO, 1997). Furlani, Bolonhesi e Fanquin (1999) salientam que muitos cultivos hidropônicos não obtêm sucesso, devido a falta de adequação da solução nutritiva e do manejo correto para a cultura a ser implantada. A obtenção da condutividade elétrica dos substratos que é a concentração inicial de sais solúveis, indica se há necessidade de repor nutrientes ou não, por meio da solução nutritiva. Já o pH influencia diretamente na disponibilidade do nutriente; substratos com $\mathrm{pH}$ próximos de 6,5 são os mais indicados (TAIZ; ZEIGER, 2006; MARTINEZ; SILVA FILHO, 1997).

O presente trabalho teve como objetivo a avaliação nutricional das plantas em função da condutividade elétrica da solução nutritiva utilizando diferentes substratos.

\section{Materiais e Métodos}

O trabalho foi conduzido no Centro de Ciências Agrárias da Universidade Estadual de Londrina,

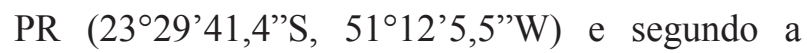
classificação de Köppen, o clima é do tipo Cfa.

O experimento utilizou a cultivar UEL-2 de feijão-vagem, que possui crescimento determinado, altura média de $40 \mathrm{~cm}$, vagens retas com secção achatada e colheita em torno de 55 dias após a emergência de acordo com Athanázio et al. (1998).
O delineamento experimental foi de blocos casualizados em parcelas subdivididas no espaço, aplicando solução nutritiva com 0,$5 ; 1,0 ; 1,5$ e $2,0 \mathrm{mS} \mathrm{cm}^{-1}$ de condutividade elétrica (CE). As subparcelas foram compostas por substrato de casca de arroz carbonizada (S.C.A.), casca de Pinus (S.C.P.) e uma mistura composta de $50 \%$ casca de arroz $+50 \%$ de casca de Pinus (S.(A+P)). Todos os tratamentos apresentaram cinco repetições, cada parcela "ou vaso" com duas plantas totalizando 60 vasos e 120 plantas.

Foram utilizados vasos de cinco litros com respectivos pratos, para retenção do eluviado e obtenção dos dados de CE e potencial hidrogeniônico $(\mathrm{pH})$. Antes da semeadura, os vasos foram equalizados em seus tratamentos conforme a CE, e adicionadas a solução de cada tratamento até se manter constante a $\mathrm{CE}$ do eluviado. Cada vaso foi deixado com duas plantas. Na tabela 1 contém os dados da composição da solução nutritiva utilizada para a elaboração dos tratamentos.

Para o preparo da solução de cada tratamento se realizou a diluição de uma solução principal (tabela 1) de $\mathrm{CE}$ de $2,3 \mathrm{mS} \mathrm{cm}^{-1}$ com a adição de água destilada até a obtenção da CE de cada tratamento, avaliando-se a CE com um condutivímetro portátil de leitura instantânea. Uma vez por semana o eluviado da fertirrigação era analisado para a obtenção dos dados de pH e CE. As fertirrigações foram realizadas manualmente e diariamente, duas vezes por dia e cada vez com $150 \mathrm{ml}$ de solução nutritiva com a utilização de um regador portátil, controlando a quantidade irrigada para que seja a mesma em todas as repetições.

As colheitas iniciaram-se quando as vagens atingiram o ponto de colheita comercial e foram repetidas até não restar mais vagens nas plantas. Foram analisadas medias de massas frescas e secas de raízes, caules, folhas e vagens, comprimento e diâmetro de vagens, CE's e pH's do eluviado durante todo ciclo, além da composição mineral do tecido vegetal. Todas as amostras de tecido após a 
coleta foram secas em estufas com circulação de ar forçada a $60^{\circ} \mathrm{C}$ até atingirem massa constante. As amostras passaram pelas digestões sulfúricas para determinação de nitrogênio e nítrico-perclórica para os demais nutrientes (MALAVOLTA, VITTI; OLIVEIRA, 1989). Os teores de nutrientes nos tecidos foram determinados com auxilio dos equipamentos denominados: Espectrofotômetro de Absorção Atômica (determinação de Cálcio, Ferro, Magnésio e Zinco), Espectrofotômetro com azul-de-molibdênio (determinação de Fósforo), Fotômetro de chama (determinação de Potássio) e Micro-destilador seguido de Titulação - Kjeldahl (determinação de Nitrogênio).

Tabela 1. Composição da solução nutritiva principal - quantidade de sais colocados em 300 litros de água, resultando em condutividade elétrica de $2,3 \mathrm{mS} \mathrm{cm}^{-1}$.

\begin{tabular}{lc}
\hline \multicolumn{1}{c}{ Sais } & g 300 \\
\hline $\mathrm{KH}_{2} \mathrm{PO}_{4}$ & 40,44 \\
NITRATO DE CÁLCIO & 278,70 \\
CLORETO DE CÁLCIO & 90 \\
SULFATO DE MAGNÉSIO & 114 \\
NITRATO DE POTÁSSIO & 120 \\
Tenso Hidro Coktail* & $900 \mathrm{~mL}$ \\
Tenso Fe & $30 \mathrm{~mL}$ \\
\hline
\end{tabular}

*Tenso Hidro Coktail ${ }^{\circledR}(2,57 ; 0,52 ; 0,13 ; 0,53 ; 2,1 ; 1,74 ; 2,57$ e 0,53\% de Ca EDTA; B; Mo; Cu EDTA; Fe EDTA; Fe DTPA; Mn EDTA e Zn EDTA respectivamente).

Fonte: Elaboração dos autores.

Os resultados obtidos foram submetidos a analise de variância e as médias dos substratos comparadas pelo teste de Tukey a 5\% de probabilidade, e as doses de condutividade elétrica por meio de regressão.

\section{Resultados e Discussões}

A maior produção de vagens foi obtida na combinação do substrato $\mathrm{S}$.(A+P) com $\mathrm{CE}$ de 1,5 $\mathrm{mS} \mathrm{cm} \mathrm{cm}^{-1}$ na solução nutritiva (tabela 2 ), atingindo valores de $182 \mathrm{~g} \mathrm{vaso}^{-1}$, correspondendo à $1,09 \mathrm{~kg}$ $\mathrm{m}^{2}$. O pior resultado foi obtido como a combinação do substrato S.C.A e com CE de $0,5 \mathrm{mS} \mathrm{cm}^{-1}$, com produção de 47,6 $\mathrm{g}$ vaso ${ }^{-1}$, sendo extrapolado para $0,286 \mathrm{~kg} \mathrm{~m}^{2}$. Moreira, Ferreira e Takahashi (2009) determinaram o potencial agronômico de genótipos de feijão-vagem, para a cultivar UEL-2, atingindo valores máximos de $0,773 \mathrm{~kg} \mathrm{~m}^{2}$ vagens, em cultivo convencional com 12 plantas $\mathrm{m}^{2}$. Observa-se que a combinação do substrato $\mathrm{S}$. $(\mathrm{A}+\mathrm{P})$ com $\mathrm{CE}$ de 1,5 $\mathrm{mS} \mathrm{cm} \mathrm{cm}^{-1}$ na solução nutritiva apresentou um ganho de cerca de $32 \%$ em relação aos valores observados por Moreira, Ferreira e Takahashi (2009) em campo.

$\mathrm{Na}$ Figura 1-a podemos observar que a medida que se aumenta a CE da solução nutritiva, a produção de feijão-vagem por vaso cresce com resposta quadrática, no entanto a partir de $1,5 \mathrm{mS} \mathrm{cm}^{-1}$ já não são observadas grandes variações, tanto para as vagens frescas quanto para as secas (figura $1 b$ ) que também apresentou uma resposta quadrática. Para essas variáveis, foi verificada interação significativa entre os substratos e as CE's. Comparando-se a média dos substratos (tabela 2) observa-se que o $\mathrm{S}$. $(\mathrm{A}+\mathrm{P})$ obteve melhores resultados de produção de vagens, pois a mistura destes substratos, promove o equilíbrio entre aeração e retenção de água, o que segundo Ansorena Miner (1994) impede o acúmulo de $\mathrm{CO}_{2}$, favorecendo a respiração celular e processo fundamental para a absorção de nutrientes. Assim com o aumento das CE's (Figura 1a e 1b) observase respostas positivas na produção. Maiores concentrações salinas consequentemente melhor 
produção, mas de acordo com Taiz e Zaiger (2004), a elevação da concentração salina (CE da solução) aumenta a capacidade produtiva até certo ponto, quando a pressão osmótica do meio, faz com que a planta encontre dificuldades em absorver água suficiente para repor a perda por transpiração, mesmo em ambientes com água disponível como é o caso da solução nutritiva.

Tabela 2. Rendimento de feijão-vagem cultivado no sistema fertirrigação.

\begin{tabular}{|c|c|c|c|c|c|c|c|}
\hline \multirow{2}{*}{ Variável } & \multirow{2}{*}{ Substrato } & \multicolumn{4}{|c|}{ Condutividade Elétrica $\left(\mathrm{mS} \mathrm{cm}^{-1}\right)$} & \multirow{2}{*}{ Média } & \multirow{2}{*}{ Regressões } \\
\hline & & $\mathbf{0 , 5 0}$ & 1,00 & 1,50 & 2,00 & & \\
\hline \multirow{5}{*}{$\begin{array}{c}\text { Vagens } \\
\text { Frescas } \\
\text { (g/vaso) }\end{array}$} & S.C.P. ${ }^{2}$ & $69,08 \mathrm{~B}^{1}$ & $139,6 \mathrm{~A}$ & $147,03 \mathrm{~A}$ & $174,28 \mathrm{~A}$ & 132,50 & * \\
\hline & S.C.A. ${ }^{3}$ & $47,6 \mathrm{C}$ & $105,75 \mathrm{~A}$ & $174,25 \mathrm{~A}$ & $178,43 \mathrm{~A}$ & 126,51 & $*$ \\
\hline & S. $(\mathrm{A}+\mathrm{P})^{4}$ & $106,18 \mathrm{~A}$ & $110,76 \mathrm{~A}$ & $181,68 \mathrm{~A}$ & $177,93 \mathrm{~A}$ & 156,79 & * \\
\hline & Média & 74,29 & 118,70 & 167,65 & 176,68 & & \\
\hline & CV & $21,25 \%$ & & & & & \\
\hline \multirow{4}{*}{$\begin{array}{c}\text { Massa } \\
\text { Seca de } \\
\text { vagens } \\
\text { (g/vaso) }\end{array}$} & S.C.P. & $4,80 \mathrm{~B}$ & $10,60 \mathrm{~A}$ & $17,30 \mathrm{~A}$ & $16,26 \mathrm{~A}$ & 12,24 & $*$ \\
\hline & S.C.A. & $4,60 \mathrm{~B}$ & $11,23 \mathrm{~A}$ & $14,28 \mathrm{~A}$ & $16,01 \mathrm{~A}$ & 11,53 & * \\
\hline & S. $(\mathbf{A}+\mathbf{P})$ & $10,75 \mathrm{~A}$ & $9,46 \mathrm{~A}$ & $13,28 \mathrm{~A}$ & $16,41 \mathrm{~A}$ & 12,48 & $*$ \\
\hline & $\begin{array}{c}\text { Média } \\
\text { CV }\end{array}$ & $\begin{array}{c}6,72 \\
22,82 \%\end{array}$ & 10,43 & 14,95 & 16,23 & & \\
\hline
\end{tabular}

${ }^{1}$ Médiasseguidas da mesma letra maiúscula nas colunas não diferem entre si pelo teste de Tukey a 5\% de probabilidade para os substratos. ${ }^{2}$ S.C.P. $=$ Substrato casca de Pinus. ${ }^{3}$ S.C.A. $=$ Substrato casca de arroz carbonizada. ${ }^{4}$ S. $(A+P)=$ Substrato arroz + pinus. *: para doses significativos; ns: para doses não significativos na análise de regressão de condutividade elétrica(CE).

Fonte: Elaboração dos autores.

$\mathrm{Na}$ tabela 3 não foi verificada diferença significativa entre os substratos para massa seca de folha, mas foi significativa a interação com a CE (figura 1c), mostrando uma resposta quadrática da $\mathrm{CE}$ em todos os substratos. Quanto os resultados de massa de plantas (tabela 3), demonstram que não houve interação entre o substrato e a CE.
Para esse parâmetro a $\mathrm{CE}$ não influenciou no desempenho dos substratos. Assim o substrato $\mathrm{S} .(\mathrm{A}+\mathrm{P})$ obteve melhores resultados no massa de plantas, independentemente da quantidade de sais fornecidas na fertirrigação. Quando se considera a média de peso de plantas de todos os substratos (figura 1- d) observa-se uma resposta positiva com o aumento da CE. 
Figura 1. Massa fresca e seca de vagens, massa seca de folhas e fresca de plantas.

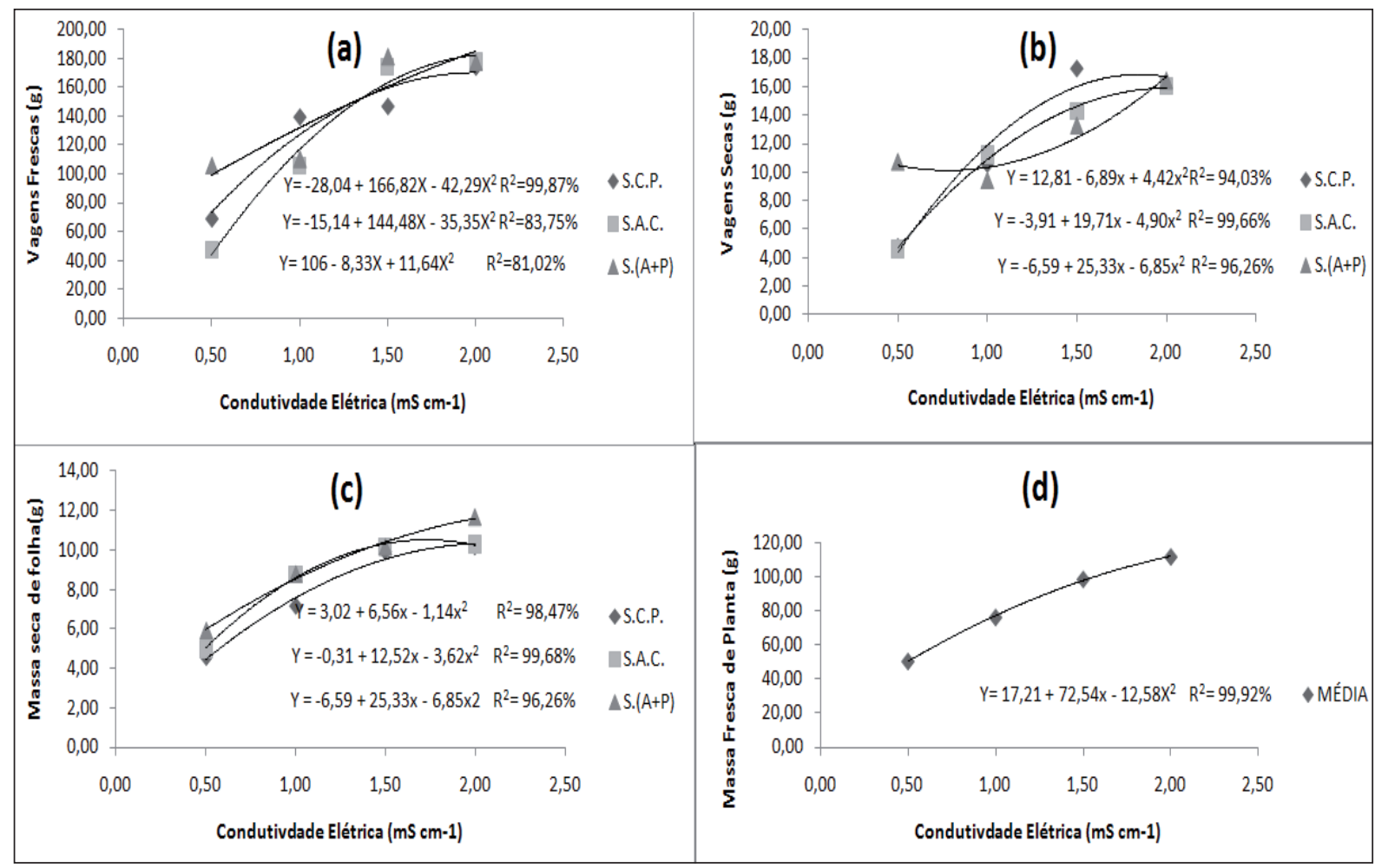

Fonte: Elaboração dos autores.

Tabela 3. Peso de plantas e Massa seca (MS) das folhas (g/vaso) de feijão-vagem cultivado com fertirrigação.

\begin{tabular}{|c|c|c|c|c|c|c|c|}
\hline \multirow{2}{*}{ Variável } & \multirow{2}{*}{ Substrato } & \multicolumn{4}{|c|}{ Condutividade Elétrica } & \multirow{2}{*}{ Média } & \multirow{2}{*}{ Regressões } \\
\hline & & 0,50 & 1,00 & 1,50 & 2,00 & & \\
\hline \multirow{5}{*}{$\begin{array}{c}\text { Massa } \\
\text { Seca de } \\
\text { Folha (g/ } \\
\text { vaso) }\end{array}$} & S.C.P. & 4,58 & 7,18 & 9,93 & 10,19 & 7,97A & $*$ \\
\hline & S.C.A. & 4,99 & 8,75 & 10,16 & 10,30 & $8,55 \mathrm{~A}$ & $*$ \\
\hline & S. $(A+P)$ & 5,89 & 8,78 & 10,16 & 11,68 & $9,13 \mathrm{~A}$ & $*$ \\
\hline & Média & 5,15 & 8,24 & 10,08 & 10,72 & & \\
\hline & $\mathbf{C V}$ & $17,87 \%$ & & & & & \\
\hline \multirow{4}{*}{$\begin{array}{l}\text { Peso de } \\
\text { Plantas } \\
\text { (g/vaso) }\end{array}$} & S.C.P. & $35,72 \mathrm{~B}$ & $65,77 \mathrm{~B}$ & $80,35 \mathrm{~B}$ & $98,25 \mathrm{~B}$ & 70,02 & ns \\
\hline & S.C.A. & $49,01 B$ & $75 \mathrm{AB}$ & $108,32 \mathrm{~A}$ & $115,15 \mathrm{~A}$ & 86,87 & ns \\
\hline & S. $(A+P)$ & $67,14 \mathrm{~A}$ & $88,13 \mathrm{~A}$ & $107,1 \mathrm{~A}$ & $121,68 \mathrm{~A}$ & 96,01 & ns \\
\hline & $\begin{array}{c}\text { Média } \\
\text { CV }\end{array}$ & $\begin{array}{l}50,62 \\
12,78 \% \\
\end{array}$ & 76,30 & 98,59 & 111,69 & & $*$ \\
\hline
\end{tabular}

${ }^{1}$ Médias seguidas da mesma letra maiúscula nas colunas não diferem entre si pelo teste de Tukey a 5\% de probabilidade para os substratos. ${ }^{2}$ S.C.P. $=$ Substrato casca de Pinus. ${ }^{3}$ S.C.A. $=$ Substrato casca de arroz carbonizada. ${ }^{4}$ S. $(A+P)=$ Substrato arroz + pinus . *: para doses significativos; ns: para doses não significativos na análise de regressão de condutividade elétrica $(\mathrm{CE})$.

Fonte: Elaboração dos autores. 
Os resultados para massa seca de raízes podem ser visualizados na tabela 4. Observa-se que

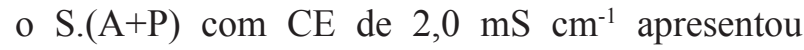
valores estatisticamente superiores em relação aos demais substratos. A partir dos resultados foi possível a constatação que a maior massa seca de raiz proporcionou as melhores produções, mas não garantiu que a melhor produção estivesse relacionada a maior massa seca de raiz. Para Medeiros, Soares e Guimarães (2005) a compactação do solo e ou substrato, provoca redução da porosidade e da aeração diminuindo drasticamente a matéria seca de raiz. Segundo Kämpf e Fermino (2000), substratos que possuem espaço de aeração superior a $42 \%$ e porosidade total acima de $80 \%$, são considerados ideais para serem utilizados em recipientes com pequeno volume. O S. $(\mathrm{A}+\mathrm{P})$ apresentou as características mais adequadas refletindo em maior produção e diferindo estatisticamente dos demais substratos. De acordo com Fachinello et al. (1995), o efeito do substrato sobre o percentual de pegamento assim como a quantidade e qualidade das raízes formadas, está especialmente relacionado à porosidade do material utilizado, que também afeta o teor da água retida, além da aeração nas raízes.

Tabela 4. Massa seca de raízes e de caules (g/vaso) de feijão-vagem cultivado com fertirrigação.

\begin{tabular}{|c|c|c|c|c|c|c|c|}
\hline \multirow{2}{*}{ Variável } & \multirow{2}{*}{ Substrato } & \multicolumn{4}{|c|}{ Condutividade Elétrica } & \multirow{2}{*}{ Média } & \multirow{2}{*}{ Regressões } \\
\hline & & $\mathbf{0 , 5 0}$ & 1,00 & 1,50 & 2,00 & & \\
\hline \multirow{5}{*}{$\begin{array}{c}\text { Massa } \\
\text { Seca de } \\
\text { Raíz (g/ } \\
\text { vaso) }\end{array}$} & S.C.P. & $7,86 \mathrm{~A}$ & $9,59 \mathrm{~A}$ & $11,41 \mathrm{~A}$ & $18,92 \mathrm{~B}$ & 11,95 & $*$ \\
\hline & S.C.A. & $6,31 \mathrm{~A}$ & $9,78 \mathrm{~A}$ & $9,99 \mathrm{~A}$ & $17,68 \mathrm{~B}$ & 10,94 & $*$ \\
\hline & S. $(A+P)$ & $6,15 \mathrm{~A}$ & $5,91 \mathrm{~A}$ & $11,87 \mathrm{~A}$ & $26,33 \mathrm{~A}$ & 12,57 & $*$ \\
\hline & Média & 6,77 & 8,43 & 11,09 & 20,98 & & \\
\hline & CV & $35,51 \%$ & & & & & \\
\hline \multirow{5}{*}{$\begin{array}{c}\text { Massa } \\
\text { Seca de } \\
\text { Caule (g/ } \\
\text { vaso) }\end{array}$} & S.C.P. & $6,07 \mathrm{~B}$ & $9,42 \mathrm{~A}$ & $10,01 \mathrm{~A}$ & $13,75 \mathrm{~A}$ & 9,81 & $*$ \\
\hline & S.C.A. & $3,68 \mathrm{~B}$ & $9,06 \mathrm{~A}$ & $10,71 \mathrm{~A}$ & $12,18 \mathrm{~A}$ & 8,91 & $*$ \\
\hline & S. $(A+P)$ & $7,49 \mathrm{~A}$ & $8,84 \mathrm{~A}$ & $13,21 \mathrm{~A}$ & $14,01 \mathrm{~A}$ & 10,89 & $*$ \\
\hline & Média & 5,75 & 9,11 & 11,31 & 13,31 & & \\
\hline & CV & $14,04 \%$ & & & & & \\
\hline
\end{tabular}

${ }^{1}$ Médias seguidas da mesma letra maiúscula nas colunas não diferem entre si pelo teste de Tukey a $5 \%$ de probabilidade para os substratos. ${ }^{2}$ S.C.P. $=$ Substrato casca de Pinus. ${ }^{3}$ S.C.A. $=$ Substrato casca de arroz carbonizada. ${ }^{4}$ S. $(A+P)=$ Substrato arroz + pinus. *: para doses significativos; ns: para doses não significativos na análise de regressão de condutividade elétrica(CE).

Fonte: Elaboração dos autores.

A massa seca de caule observada na tabela 4, demonstra que o $\mathrm{S} .(\mathrm{A}+\mathrm{P})$ foi superior

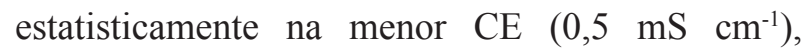
mas em concentrações acima de $0,5 \mathrm{mS} \mathrm{cm}^{-1}$ os substratos apresentaram estatisticamente o mesmo comportamento. Já para massa seca de raízes, não são observadas diferenças significativas com as CE's de 0,$5 ; 1,0$ e $1,5 \mathrm{mS} \mathrm{cm}^{-1}$, independente do tipo de substrato. Entretanto, com CE de 2,0 mS $\mathrm{cm}^{-1}$ o S. $(\mathrm{A}+\mathrm{P})$ demonstrou ser o melhor tratamento para o crescimento de raízes. Relacionando as CE's tanto para massa seca de raiz (Figura 2a) quanto massa seca de caule (Figura 2b) podemos observar interações significativas, positivas e respostas quadráticas com o aumento da $\mathrm{CE}$ dos tratamentos, resultando em crescente aumento das massas secas de raiz e caule. 
Figura 2. Massa seca de raiz e caule, diâmetro e número de vagens.

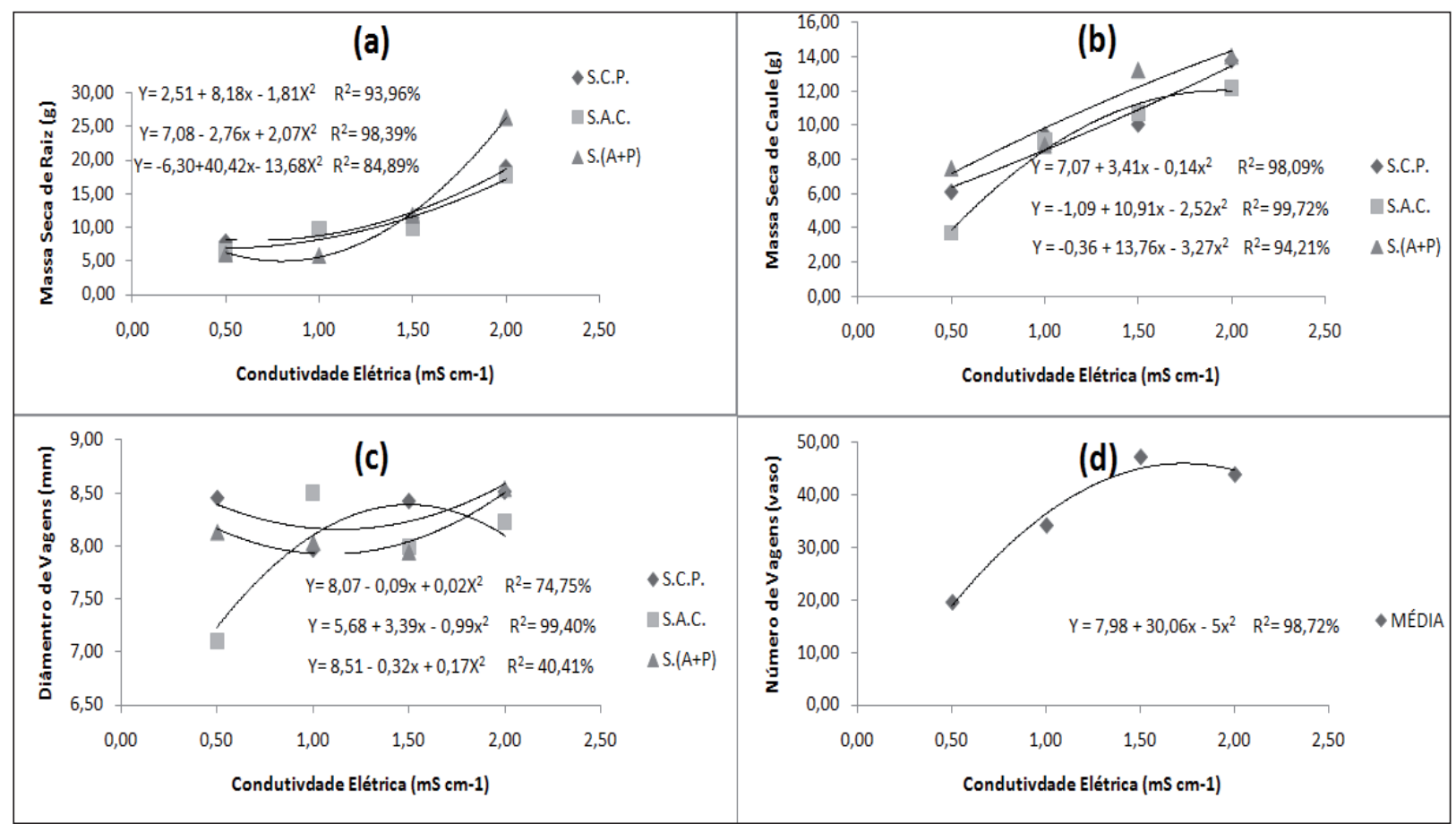

Fonte: Elaboração dos autores.

Na Figura 3 podem ser visualizadas as médias dos pH's dos substratos durante a realização do experimento. O S.C.A. manteve seu $\mathrm{pH}$ entre 6 a 6,5. Já o S.C.P., inicialmente manteve o $\mathrm{pH}$ ácido $(4,0)$, mas conforme o desenvolvimento da cultura e a utilização da fertirrigação com solução nutritiva de $\mathrm{pH}$ 6,3, sua acidez foi reduzindo, alcançando $\mathrm{pH}$ de 5,9, nível considerado adequado para a maioria das culturas. Assim a utilização antecipada da solução nutritiva neste substrato reduziria a sua acidez e a cultura seria implantada em um meio mais adequado. Outra opção seria a mistura com um substrato que apresente $\mathrm{pH}$ mais elevado, como o S.C.A. que manteve o $\mathrm{pH}$ em níveis proóximos da neutralidade, o que segundo Minami (2000) é de fundamental importância, pois facilitam a absorção de nutrientes devido ao aumento da disponibilidade. Segundo Ansorena Miner (1994) as propriedades físicas de um substrato não podem ser modificadas já que são relacionadas ao material utilizado, já as características químicas podem ser alteradas mediante irrigações e adubações.

Os resultados de $\mathrm{pH}$ dos substratos em cada CE podem ser observados na figura 3 . Nota-se que o tratamento de $2,0 \mathrm{mS} \mathrm{cm}^{-1}$ influenciou muito mais o aumento do $\mathrm{pH}$ no S.C.P., quando comparado com os tratamentos com menores CE e também em relação aos outros substratos com mesma condutividade. Com a adição de solução com $\mathrm{CE}$ de $2 \mathrm{mS} \mathrm{cm}^{-1}$, as concentrações de bases como cálcio, magnésio e potássio se tornaram maiores, provocando o aumento do $\mathrm{pH}$. 
Figura 3. Médias dos pH's dos substratos conforme as leituras semanais.

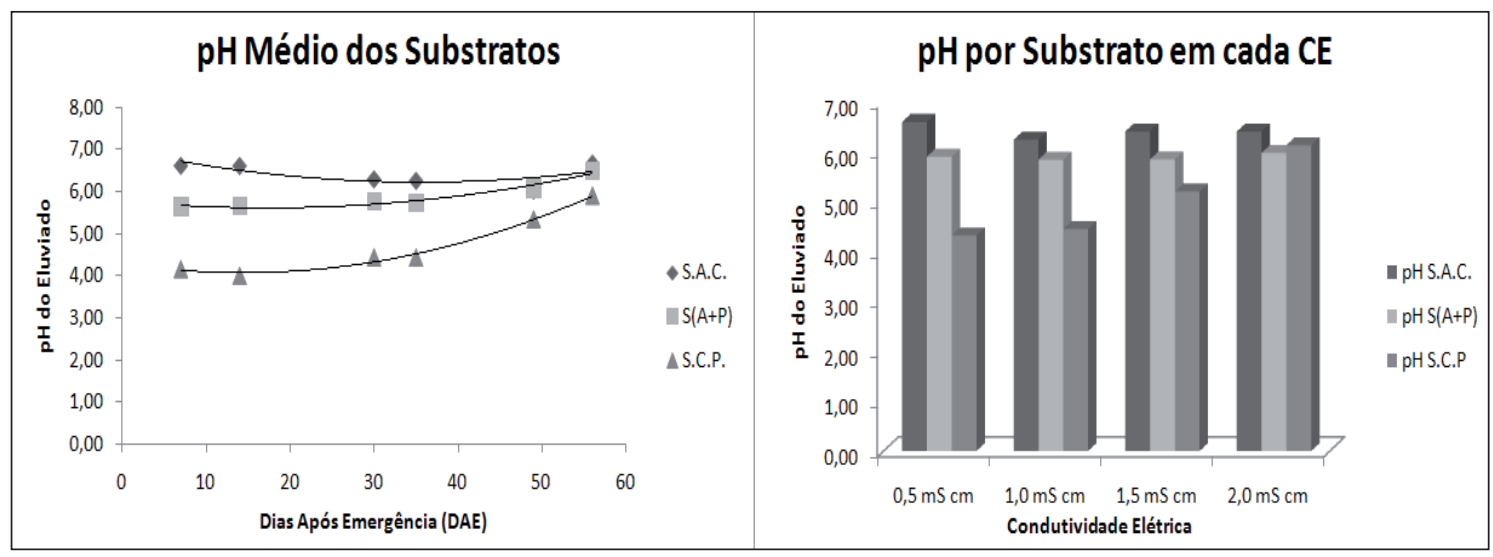

Fonte: Elaboração dos autores.

A partir das leituras de $\mathrm{CE}$ do eluviado (Figura 3), se observou que do inicio de plantio até o enchimento das vagens, por volta dos 40 DAE, as concentrações de sais nos eluviados que eram elevadas (colocar aqui o valor), foram diminuindo até um determinado até o fim do enchimento de vagens (colocar aqui o valor), e depois retomaram o crescimento da $\mathrm{CE}$, demonstrando que a planta necessita de nutrientes em quantidades variadas conforme o seu desenvolvimento (MARTINEZ; SILVA FILHO, 1997). Na quarta leitura (35 DAE) a planta se encontrava em início de florescimento, período em que a alta disponibilidade de nutrientes é essencial para formação das vagens. E mesmo com o fornecimento constante de solução nutritiva conforme os tratamentos utilizados, não foi observado aumento da CE no eluviado. As CE's dos eluviados apresentaram um comportamento semelhante independente da CE utilizada. Assim plantas desenvolvidas com $\mathrm{CE}$ altas (1,5 e 2,0 mS $\mathrm{cm}^{-1}$ ), obtiveram maiores necessidades nutricionais e melhores potenciais de absorção, do que plantas desenvolvidas com baixa CE $\left(0,5\right.$ e $1,0 \mathrm{mS} \mathrm{cm} \mathrm{cm}^{-}$ $\left.{ }^{1}\right)$, resultando em um equilíbrio neste momento entre as CE's dos eluviados. Na ultima leitura foi observado aumento da CE dos eluviados, pois as plantas atingiram a maturação fisiológica, reduzindo o consumo de nutrientes até chegar ao fim do ciclo

Figura 4. Médias das condutividades elétricas dos eluviados.

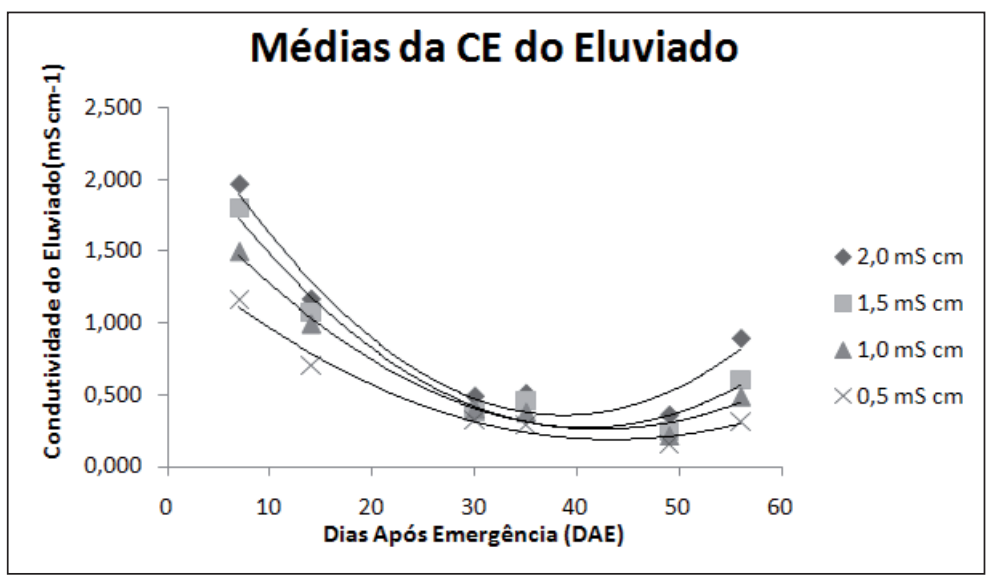

Fonte: Elaboração dos autores. 
Nas tabelas 5 e 6 podem ser observados os teores de nitrogênio, potássio, fósforo e cálcio nas vagens. Não foram detectadas grandes variações em relação aos nutrientes (N, K e P) nos diferentes tratamentos. Nota-se que o S.(A+P), com CE baixa $(0,5 \mathrm{mS}$ $\mathrm{cm}^{-1}$ ) foi superior estatisticamente em relação os demais substratos, tanto para nitrogênio quanto para potássio, porém com o aumento da CE $(1,0$; 1,5 e $2 \mathrm{mS} \mathrm{cm}^{-1}$ ) os substratos não se diferenciaram estatisticamente quanto aos teores de nutrientes na vagem. O teor de nitrogênio, potássio e de fósforo nas folhas (figura $5 \mathrm{a}, \mathrm{b}$ e c respectivamente) apresentaram respostas quadráticas com o aumento da CE da solução nutritiva e interação significativa do substrato com a CE.
Com relação ao teor de cálcio $\left(\mathrm{mg} \mathrm{vaso}^{-1}\right)$ na massa seca de vagens (tabela 6), pode-se observar diferenças significativas entre os substratos. OS.C.A. apresentou melhores resultados para o teor de cálcio em relação aos outros substratos. Entretanto para as $\mathrm{CE}\left(0,5\right.$ e $\left.2,0 \mathrm{mS} \mathrm{cm}^{-1}\right)$ o $\mathrm{S} .(\mathrm{A}+\mathrm{P})$ apresentou o mesmo comportamento que o S.C.A., concordando com resultados obtidos por Minami (1995), que também observou maior disponibilidade de cálcio para as plantas com a utilização do substrato casca de arroz. Os teores de cálcio aumentaram com respostas quadráticas conforme a $\mathrm{CE}$ foi elevada (figura $3 \mathrm{c}$ e d).

Tabela 5. Teor de nitrogênio e potássio em vagens de plantas cultivadas com fertirrigação.

\begin{tabular}{cccccccc}
\hline \multirow{2}{*}{ Variável } & Substrato & \multicolumn{9}{c}{ Condutividade Elétrica } & \multirow{2}{*}{ Média } & \multirow{2}{*}{ Regressões } \\
\cline { 3 - 6 } & & $\mathbf{0 , 5 0}$ & $\mathbf{1 , 0 0}$ & $\mathbf{1 , 5 0}$ & $\mathbf{2 , 0 0}$ & & $*$ \\
S.C.P. & $92,87 \mathrm{~B}$ & $226,35 \mathrm{~A}$ & $360,51 \mathrm{~A}$ & $401,95 \mathrm{~A}$ & 270,42 & $*$ \\
Nitrogênio & S.C.A. & $119,30 \mathrm{~B}$ & $301,01 \mathrm{~A}$ & $378,31 \mathrm{~A}$ & $409,10 \mathrm{~A}$ & 301,93 & $*$ \\
& S.(A+P) & $257,83 \mathrm{~A}$ & $239,66 \mathrm{~A}$ & $311,42 \mathrm{~A}$ & $415,15 \mathrm{~A}$ & 306,02 & $*$ \\
\cline { 2 - 6 } & Média & 156,67 & 255,67 & 350,08 & 408,73 & & \\
Teor de & CV & $22,01 \%$ & & & & & $*$ \\
Potássio & S.C.P. & $120,63 \mathrm{~B}$ & $285,47 \mathrm{~A}$ & $510,15 \mathrm{~A}$ & $448,84 \mathrm{~A}$ & 341,27 & $*$ \\
& S.C.A. & $142,18 \mathrm{~B}$ & $345,50 \mathrm{~A}$ & $422,12 \mathrm{~A}$ & $483,41 \mathrm{~A}$ & 348,30 & $*$ \\
\cline { 2 - 6 } & S.(A+P) & $343,61 \mathrm{~A}$ & $293,52 \mathrm{~A}$ & $399,18 \mathrm{~A}$ & $495,11 \mathrm{~A}$ & 382,86 & $*$ \\
\hline
\end{tabular}

\footnotetext{
${ }^{1}$ Médias seguidas da mesma letra maiúscula nas colunas não diferem entre si pelo teste de Tukey a $5 \%$ de probabilidade para os substratos. ${ }^{2}$ S.C.P. $=$ Substrato casca de Pinus. ${ }^{3}$ S.C.A. $=$ Substrato casca de arroz carbonizada. ${ }^{4}$ S. $(A+P)=$ Substrato arroz + pinus. *: para doses significativos; ns: para doses não significativos na análise de regressão de condutividade elétrica(CE).

Fonte: Elaboração dos autores.
} 
Figura 5. Teores de Nutrientes nas Vagens.

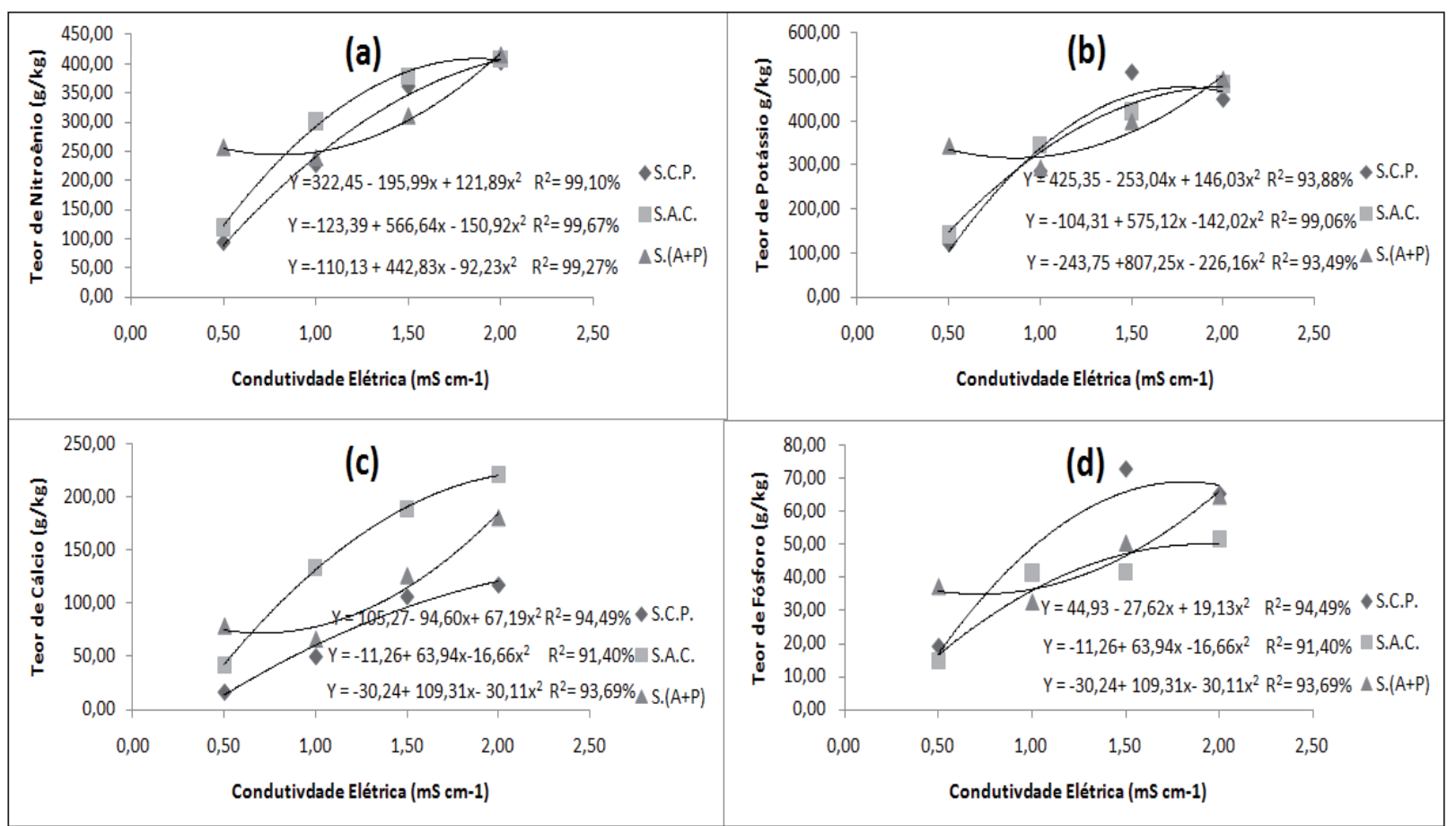

Fonte: Elaboração dos autores.

Tabela 6. Teor de Fosforo e cálcio em vagens de plantas cultivadas com fertirrigação.

\begin{tabular}{|c|c|c|c|c|c|c|c|}
\hline \multirow{2}{*}{ Variável } & \multirow{2}{*}{ Substrato } & \multicolumn{4}{|c|}{ Condutividade Elétrica } & \multirow{2}{*}{ Média } & \multirow{2}{*}{ Regressões } \\
\hline & & $\mathbf{0 , 5 0}$ & 1,00 & 1,50 & 2,00 & & \\
\hline \multirow{5}{*}{$\begin{array}{l}\text { Teor de } \\
\text { Cálcio }\end{array}$} & S.C.P. & $17,1 \mathrm{~B}$ & $50,31 \mathrm{~B}$ & $106,39 \mathrm{~B}$ & $117,37 \mathrm{~B}$ & 72,79 & $*$ \\
\hline & S.C.A. & $41,78 \mathrm{AB}$ & $133,35 \mathrm{~A}$ & $188,48 \mathrm{~A}$ & $220,83 \mathrm{~A}$ & 146,11 & $*$ \\
\hline & S. $(\mathrm{A}+\mathrm{P})$ & $78,69 \mathrm{~A}$ & $66,11 \mathrm{~B}$ & $126,3 \mathrm{~B}$ & $180,91 \mathrm{~A}$ & 113,00 & $*$ \\
\hline & Média & 45,86 & 83,26 & 140,39 & 173,04 & & \\
\hline & $\mathrm{CV}$ & $32,61 \%$ & & & & & \\
\hline \multirow{5}{*}{$\begin{array}{l}\text { Teor de } \\
\text { Fóforo }\end{array}$} & S.C.P. & $19,26 \mathrm{~B}$ & $41,84 \mathrm{~A}$ & $73,10 \mathrm{~A}$ & $65,57 \mathrm{~A}$ & 49,94 & $*$ \\
\hline & S.C.A. & $14,71 \mathrm{~B}$ & $41,51 \mathrm{~A}$ & $41,66 \mathrm{~B}$ & $51,80 \mathrm{~A}$ & 37,42 & $*$ \\
\hline & S. $(\mathrm{A}+\mathrm{P})$ & $37,22 \mathrm{~A}$ & $32,46 \mathrm{~A}$ & $50,51 \mathrm{~B}$ & $64,88 \mathrm{~A}$ & 46,27 & $*$ \\
\hline & Média & 23,73 & 38,60 & 55,09 & 60,75 & & \\
\hline & $\mathrm{CV}$ & $22,74 \%$ & & & & & \\
\hline
\end{tabular}

${ }^{1}$ Médias seguidas da mesma letra maiúscula nas colunas não diferem entre si pelo teste de Tukey a 5\% de probabilidade para os substratos. ${ }^{2}$ S.C.P. $=$ Substrato casca de Pinus. ${ }^{3}$ S.C.A. $=$ Substrato casca de arroz carbonizada. ${ }^{4}$ S. $($ A + P $)=$ Substrato arroz + pinus. *: para doses significativos; ns: para doses não significativos na análise de regressão de condutividade elétrica $(\mathrm{CE})$.

Fonte: Elaboração dos autores. 
As características de diâmetro, número e comprimento de vagens, são representadas na tabela 7. Os melhores resultados para o diâmetro foram obtidos conforme o aumento da CE, apresentando interação significativa com os substratos e resposta quadrática (figura $2 \mathrm{c}$ ). Considerando as médias dos substratos para esta variável, somente com CE de $0,5 \mathrm{mS} \mathrm{cm}^{-1}$, o S.C.A. apresentou o pior resultados quando comparados com os outros substratos, porém em CEs superiores, essa diferença não foi detectada. Para o numero de vagens e comprimento médio de vagens não foi observada interação do substrato com a CE, ou seja, o substrato foi responsável pela diferença significativa verificada nos resultados. O maior número de vagens por vaso foi obtido com a utilização do $\mathrm{S}$.(A+P) para as $\mathrm{CE}$

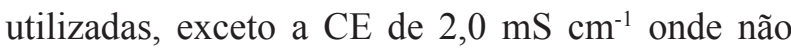
foi observada diferença significativa em relação aos demais substratos. Quanto ao comprimento de vagens, o S.C.A. foi superior estatisticamente, independentemente da CE da solução aplicada.

Tabela 7. Diâmetro, número e comprimento de vagens de plantas cultivadas com fertirrigação.

\begin{tabular}{|c|c|c|c|c|c|c|c|}
\hline \multirow{2}{*}{ Variável } & \multirow{2}{*}{ Substrato } & \multicolumn{4}{|c|}{ Condutividade Elétrica } & \multirow{2}{*}{ Média } & \multirow{2}{*}{ Regressões } \\
\hline & & 0,50 & 1,00 & 1,50 & 2,00 & & \\
\hline \multirow{5}{*}{$\begin{array}{l}\text { Diâmetro de } \\
\text { vagens (média } \\
\text { /vaso) }\end{array}$} & S.C.P. & $8,46 \mathrm{~A}$ & $7,97 \mathrm{~A}$ & $8,43 \mathrm{~A}$ & $8,52 \mathrm{~A}$ & 8,35 & * \\
\hline & S.C.A. & $7,10 \mathrm{~B}$ & $8,50 \mathrm{~A}$ & $7,99 \mathrm{~A}$ & $8,23 \mathrm{~A}$ & 7,96 & $*$ \\
\hline & S. $(A+P)$ & $8,13 \mathrm{~A}$ & $8,03 \mathrm{~A}$ & $7,94 \mathrm{~B}$ & $8,54 \mathrm{~A}$ & 8,16 & $*$ \\
\hline & Média & 7,90 & 8,17 & 8,12 & 8,43 & & \\
\hline & CV & $3,65 \%$ & & & & & \\
\hline \multirow{4}{*}{$\begin{array}{l}\text { Número de } \\
\text { Vagens por } \\
\text { vaso }\end{array}$} & S.C.P. & $18,00 \mathrm{~B}$ & $33,40 \mathrm{~B}$ & $35,20 \mathrm{~B}$ & $43,80 \mathrm{~A}$ & 32,60 & ns \\
\hline & S.C.A. & $15,40 \mathrm{~B}$ & $27,40 \mathrm{~B}$ & $51,20 \mathrm{AB}$ & $44,00 \mathrm{~A}$ & 34,50 & ns \\
\hline & S. $(A+P)$ & $25,26 \mathrm{~A}$ & $41,60 \mathrm{~A}$ & $55,00 \mathrm{~A}$ & $43,60 \mathrm{~A}$ & 41,37 & $\mathrm{~ns}$ \\
\hline & $\begin{array}{c}\text { Média } \\
\text { CV }\end{array}$ & $\begin{array}{c}19,55 \\
18,18 \% \\
\end{array}$ & 34,13 & 47,13 & 43,80 & & $*$ \\
\hline \multirow{4}{*}{$\begin{array}{c}\text { Comprimento } \\
\text { Médio de } \\
\text { vagens }\end{array}$} & S.C.P. & $8,96 \mathrm{~A}$ & $9,04 \mathrm{~B}$ & $9,45 \mathrm{~B}$ & $9,23 \mathrm{~A}$ & 9,17 & ns \\
\hline & S.C.A. & $9,53 \mathrm{~A}$ & $10,36 \mathrm{~A}$ & $11,03 \mathrm{~A}$ & $10,03 \mathrm{~A}$ & 10,24 & ns \\
\hline & S. $(A+P)$ & $8,82 \mathrm{~A}$ & $8,99 \mathrm{AB}$ & $9,02 \mathrm{~B}$ & $8,83 \mathrm{~A}$ & 8,92 & $\mathrm{~ns}$ \\
\hline & $\begin{array}{l}\text { Média } \\
\text { CV }\end{array}$ & $\begin{array}{l}9,10 \\
9,42 \%\end{array}$ & 9,46 & 9,83 & 9,36 & & ns \\
\hline
\end{tabular}

${ }^{1}$ Médias seguidas da mesma letra maiúscula nas colunas não diferem entre si pelo teste de Tukey a 5\% de probabilidade para os substratos. ${ }^{2}$ S.C.P. $=$ Substrato casca de Pinus. ${ }^{3}$ S.C.A. $=$ Substrato casca de arroz carbonizada. ${ }^{4}$ S. $(A+P)=$ Substrato arroz + pinus . *: para doses significativos; ns: para doses não significativos na análise de regressão de condutividade elétrica $(\mathrm{CE})$.

Fonte: Elaboração dos autores.

\section{Conclusões}

O substrato com a mistura de casca de arroz carbonizada + casca de pínus, mostrouse interessante, pois apresenta alguns resultados superiores em comparação aos substratos utilizados de forma isolada por apresentarem características químicas mais adequadas ao desenvolvimento das plantas.

Dentre os tratamentos analisados, relacionados a nutrição das plantas, os melhores resultados foram obtidos com a utilização das maiores condutividades elétricas, entre 1,5 e $2,0 \mathrm{mS} \mathrm{cm}^{-1}$.

Para a produção de vagens frescas, tanto os tratamentos 1,5 e $2,0 \mathrm{mS} \mathrm{cm} \mathrm{cm}^{-1}$ com qualquer um dos três substratos, demonstram ser adequados em termos produtivos $\left(\mathrm{g} \mathrm{m}^{2}\right)$.

A utilização de casca de arroz como substrato se mostrou interessante pelo fornecimento de cálcio para as plantas. 


\section{Referências}

ANSORENA MINER, J. Substratos - propriedades $e$ caracterização. Madrid: Mundi-Prensa, 1994. 172 p.

ATHANÁZIO, J. C.; TAKAHASHI, L. S. A.; ENDO, R. M.; SILVA, G. L. da. "UEL-2": cultivar de feijãode-vagem tipo manteiga de crescimento determinado. Horticultura Brasileira, Brasília, v. 16, n. 1, p. 91, maio 1998.

FACHINELLO, J. C.; HOFFMAN, A.; NACHTIGAL, J. C.; KERSTEN, E.; FORTES, G. R. L. Propagação de plantas frutiferas de clima temperado. 2. ed. Pelotas: UFPel, 1995. 178 p.

FERNANDES, C.; CORÁ, J. E.; ARAÚJO, J. A. C. de. Teores foliares de nutrientes em tomateiro cultivado em substrato com parcelamento da fertirrigação. In: WORKSHOP DE FERTIRRIGAÇÃO, 2., 2001, Piracicaba. Anais.... Piracicaba: LER/ESALQ/USP, 2001. p. 201-210.

FILGUEIRA, F. A. R. Novo Manual de olericultura: agrotecnologia moderna na produção e comercialização de hortaliças. Viçosa: UFV, 2000. 402 p.

FURLANI, P. R.; BOLONHESI, L. C. P.; FANQUIN, V. Cultivo hidropônico de plantas. Campinas: Instituto Agronômico, 1999. 52 p. (Boletim técnico, 180).

GONÇALVES, J. L. M.; BENEDETTI, V. Reflexos do cultivo mínimo e intensivo do solo em sua fertilidade e na nutrição das árvores. In: GONÇALVES, J. L. M.; STAPE, J. L.; BENEDETTI, V.; FESSEL, V. A. G.; GAVA, J. L. Nutrição e fertilização florestal. Piracicaba: Instituto de Pesquisas e Estudos Florestais, 2000. p. 309350.

INSTITUTO BRASILEIRO DE GEOGRAFIA E ESTATÍSTICA - IBGE. Censo agropecuário de 2006. Rio de Janeiro: IBGE, 2006. p. 396.

KÄMPF, A. N.; FERMINO, M. H. (Ed.). Substrato para plantas: a base da produção vegetal em recipientes. Porto Alegre: Gênesis, 2000. p. 209-215.

MALAVOLTA, E.; VITTI, G. C.; OLIVEIRA, S. A. Avaliação do estado nutricional de plantas. Piracicaba: Potafos, 1989. p. 201.
MARTIN, T. N.; LIMA, L. B.; RODRIGUES, A.; GIRARDI, E.; FABRI, E. G.; MINAMI, K. Utilização da vermiculita, casca de pínus e carvão na produção de mudas de pepino e de pimentão. Acta Scientiarum Agronomy, Maringá, v. 28, n. 1, p. 107-113, 2006.

MARTINEZ, H. E. P.; BARBOSA, G. J. Substratos para hidroponia. Informe Agropecuário, Belo Horizonte, n. 20, p. 81-89, 1999.

MARTINEZ, H. E. P.; SILVA FILHO, J. B. Introdução ao cultivo hidropônico de plantas. Viçosa: UFV, 1997. $52 \mathrm{p}$.

MEDEIROS, R. D.; SOARES, A. A.; GUIMARÃES, R. M. Compactação do solo e manejo da água. I: efeitos sobre absorção de N, P, K, massa seca de raízes e parte aérea de plantas de arroz. Ciência e Agrotecnologia, Lavras, v. 29, n. 5, p. 940-947, 2005.

MINAMI, K. Adubação em substrato. In: KÄMPF, A. N.; FERMINO, M. H. (Ed.). Substrato para plantas: base da produção vegetal em recipientes. Porto Alegre: Genesis, 2000. p. 147-152.

- Produção de mudas de alta qualidade em horticultura. São Paulo: T. A. Queiroz, 1995. 128 p.

MOREIRA, R. M. P.; FERREIRA, J. M.; TAKAHASHI, L. S. A. Potencial agronômico e divergência genética entre genótipos de feijão-vagem de crescimento determinado. Semina: Ciências Agrárias, Londrina, v. 30, p. 1051-1060, 2009. Suplemento 1.

RODRIGUES, L. R. F. Cultivo pela técnica de hidroponia. Técnicas de cultivo hidropônico e de controle ambiental no manejo de pragas, doenças e nutrição vegetal em ambiente protegido. Jaboticabal: Funep, 2002. 726 p.

SIMMLER, A. O.; BLUM, L. E. D.; KOTHE, D. M.; GUIMARÃES, L. S.; PRADO, G. Efeito da casca de pínus no teor de nutrientes e no $\mathrm{pH}$ do solo. In: JORNADA ACADÊMICA, 4., 1999, Florianópolis. Anais... Florianópolis: UDESC, 1999, p. 80.

TAIZ, L.; ZEIGER, E. Minerais em excesso limitam o crescimento das plantas. In: Fisiologia vegetal. Porto Alegre: Artmed, 2004. v. 3, cap. 5, p. 100-111.

- Soluções nutritivas e rápido crescimento vegetal. Fisiologia vegetal. 3. ed. Porto Alegre: Artmed, 2004. $719 \mathrm{p}$. 
